\title{
Три подхода к определению понятий на основе собственных свойств модели
}

\author{
С.В. Микони \\ Санкт-Петербургский институт информатики и автоматизации РАН \\ smikoni@mail.ru
}

\section{Аннотация}

Предлагается применение системного подхода к анализу определения понятия. Оно рассматривается как текстовая модель, к которой применимы собственные свойства модели. Эти свойства выводятся из модели языка предикатов первого порядка, каковой является алгебраическая структура. К собственным свойствам модели отнесены функция объекта, реализующая её операция и структура. Каждое свойство представляется элементарной моделью: функциональной (Ф-модель), операционной (О-модель) и структурной (С-модель) моделью.

Использование этих моделей для анализа определения понятия названо соответственно функциональным, операционным и структурным подходом. Сущность каждого подхода поясняется на примере определения термина «менеджмент качества», взятого из международного терминологического стандарта. Для иллюстрации подходов применяются наглядные модели графов.

Структурный подход позволяет найти избыточные и недостающие поясняющие слова в определения понятия, представленного в форме повествовательного предложения. Функциональный подход позволяет оценить полноту содержания понятия и наличие порочных циклов. Операционный подход уточняет понятия, относящиеся к категории действия, через элементарные действия.

Ключевые слова: слово, смысл, понятие, модель, свойство, базис, функция, операция, структура, отношение, аргумент.

Библиографическая ссылка: Микони С.В. Три подхода к определению понятий на основе собственных свойств модели // Компьютерная лингвистика и вычислительные онтологии. Выпуск 4 (Труды XXIII Международной объединенной научной конференции «Интернет и современное общество», IMS-2020, Санкт-Петербург, 17 20 июня 2020 г. Сборник научных статей). - СПб: Университет ИТМО, 2020. C. 109-117. DOI: 10.17586/0000-0000-2020-4-109-117

\section{Введение}

Человеческий мозг оперирует моделями. Универсальной системой моделирования внешнего и внутреннего мира человека является естественный язык (ЕЯ) [1]. Обозначая некоторый класс сущностей, каждое слово (знак) обозначает частную модель ЕЯ. В процессе углубления знаний о Природе и творении искусственного мира появляются новые сущности и потребность в их обозначении [2]. Возникающие для обмена профессиональными знаниями языки регламентируются терминологическими стандартами. Они разрабатываются специалистами предметной области (ПрО), как правило, имеющими неглубокие познания в языкознании. Роль профессиональных лингвистов ограничивается анализом этих документов на предмет соблюдения правил грамматики без углубления в профессиональный смысл текстов. На практике это влечёт невысокое качество текстового представления профессиональных знаний. 
По опыту участия в рабочей группе при разработке терминологического стандарта по технической диагностике [3] автор, следуя Д.С. Лотте [4], считает, что определение термина носит договорной характер. А это - простор для эвристики, когда под давлением интересов наиболее влиятельной группы принимается её точка зрения. Согласно Гёделю аргументами в споре может быть только внешнее (мета) знание, поскольку «непротиворечивость аксиом арифметики нельзя доказать, исходя из самих аксиом арифметики» [5]. Руководствуясь этим принципом, автор предложил язык для определения понятий и формальный подход к порождению и делению понятий на основе теоретико-множественного представления их содержания и объёма [6]. Этот подход был применён при разработке стандарта [3].

В настоящей работе определение термина рассматривается с точки зрения системного подхода. В соответствии с ним оно, как повествовательное предложение, относится к классу онтологических моделей, к которым применимы собственные свойства модели, предложенные в работе [7]. Покажем, как их можно использовать для оценивания и улучшения качества определений понятий. Согласно системному подходу, они должны удовлетворять требованиям полноты, неизбыточности, непротиворечивости и ясности.

\section{1. Базис элементарных моделей}

В работе [7] было доказано, что вся необходимая информация для формирования обоснованного базиса элементарных свойств модели содержится в модели языка предикатов первого порядка. Предика́т (лат. praedicatum - сказанное) - языковое выражсение, обозначающее какое-либо свойство или отношение. Иными словами, язык, как средство мышления первичен. Предикат - это и есть первичная модель некоторой стороны окружающего мира. Самых общих предикатов всего два: быmb (to be) и делать (to do). Недаром эти два глагола играют роль вспомогательных в английском языке. Они и приходят на помощь при формировании любых предложений независимо от их смысла.

Отметим два важных свойства этих глаголов. На основе глагола Быть формируются атрибутивные отношения (объект есть быстрый, экономичный и т.п.). На основе переходного глагола делать формируется субъект-объектное отношение (кто делает что). Отсюда следуют одноместный предикат Быmb $(x)$ и двухместный предикат Делать $(x, y)$. Предикат Быть $(x)$ обладает только унарной связью - рефлексией на себя, а предикат Делать $(x, y)$ - бинарной связью (Субъект, Объект).

Произвольное множество предикатов $P$ входит в состав многосортной алгебраической системы (структуры), представляемой четверкой множеств [8]:

$$
<A, C, F, P>\text {, }
$$

где: $A$ - множество предметных переменных (носитель), $C$ - множество констант и $F-$ множество функций, $P-$ множество предикатов. Согласно [9], многосортная алгебраическая система является моделью языка предикатов первого порядка $\Omega$. Ее элементы - суть результаты интерпретации этого языка. Пару $<F, R>$ называют сигнатурой системы. Использование только одного из этих символов представляют собой частные случаи алгебраической системы. К ним относятся соответственно алгебра

$\mathbf{A}=\langle A, C, F>$ и реляционная система (модель) $\mathbf{B}=\langle A, C, R>[9]$.

Любая функция $f \in F$ в алгебре $=\langle A, C, F\rangle$ представляет собой отображение области её определения $X, X \subset C$, в область значений $Y, Y \subset C$.

Согласно теоретико-множественному представлению функции $f: X \times \ldots \times X \rightarrow Y$ отображение области её определения в область значений («чёрный ящик») можно рассматривать и как функцию, и как операцию. За функиию примем ожидаемое отображение «входы-выходы» рассматриваемого элемента («чёрного ящика»). Оно представляется таблицей соответствия входных и выходных состояний (векторов) этого элемента. По имени известной функции можно установить результат отображения $f$. За операцию принимается процесс получения этого соответствия. 
В модельной интерпретации функция представляется функциональной моделью (Ф-моделью), а операция - операционной моделью $(\boldsymbol{O}$-моделью). Ф-модель играет роль носителя О-модели. Входы и выходы Ф-модели - физические, а входы и выходы О-модели - информационные (вещественные, энергетические).

Структурная модель наследует реляционную систему $\mathbf{B}$, отражая связи между элементами системы. Элементы с именами из множества $C$ принадлежат носителю $A$, а связи между элементами $a \in A$ принадлежат отношению смежности $R_{\mathrm{c}} \subseteq A \times A$ :

$$
M_{\mathrm{c}}=<A, C, R_{\mathrm{c}}>\text {. }
$$

При интерпретации символов $A$ и $R_{\text {c }}$ реляционной системы множествами вершин $V$ и

связей $E$ между ними (рёбер и/или дуг) переходим к модели графа $G=(V, C, E)$. Граф является наглядной моделью любой структуры $(\boldsymbol{C}$-моделью $)$.

При рассмотрении элемента как системы выполняется раскрытие его содержимого через взаимосвязь реализующих его функций и операций, играющих роль элементов. Этому соответствует переход от Ф-модели элемента к структурно-функциональной модели системы (СФ-модели), а О-модели элемента к структурно-операционной модели (О-модели). СФ-модель показывает устройство системы (раскрытого «чёрного ящика») и играет роль носителя выполняемого процесса, а СО-модель отражает процесс использования элементов носителя для получения результата. СФ- и СО-модели системы, реализующей одну функцию исходного элемента («чёрного ящика»), совпадают.

В работе [7] показано, что функциональная, операционная и структурная составляющие $<$ Функция, Операция, Структура > присущи любой модели, что позволяет отнести их к её собственным свойствам. Поскольку Ф-, О- и С-модели образуют базис собственных свойств, они применимы для анализа моделей любого типа. Воспользуемся этими свойствами применительно к таким лингвистическим моделям как определения понятий. Подход к анализу определения понятия с применением каждой из элементарных моделей будем называть её именем.

\section{2. Структурный подход}

Структурный подход основан на упорядочении слов определения понятия в отношении пояснения. Первым кандидатом на пояснение является родовое понятие, имеющее большую категорию общности, чем определяемый термин. Все остальные слова определения используются для поэтапной конкретизации смысла доминирующего слова. Конкретизация смысла очередного слова осуществляется путём задания соответствующих вопросов к поясняющим его словам.

Каждое поясняющее слово принимается за аргумент поясняемого слова, выделяемый скобками. Скобочная форма определения понятия строится путём синтаксического анализа повествовательного предложения последовательным выделением поясняющих слов с использованием падежей и предлогов. Строго говоря, скобочная модель определения понятия является СФ-моделью, поскольку каждое поясняющее слово представимо Ф-моделью.

В качестве примера построим скобочную СФ-модель определения термина «менеджмент качества». В международном стандарте [10] он определён как: скоординированная деятельность по руководству и управлению организацией применительно к качеству.

Переведя все поясняющие слова в именительный падеж, и заключив их в скобки, получим следующую аналитическую формулу определения понятия «менеджмент качества»: Менеджмент качества = (Деятельность (Скоординированная, Руководство (Организачия, Качество), Управление (Организация, Качество)).

Поясняемое слово в скобочной форме выделено жирным шрифтом. Оно само является поясняющим по отношению к определяемому термину. Полученная скобочная форма представляет собой аналитическую запись определения понятия, представимую графом 
типа «дерево». Каждая ветвь дерева сверху именуется поясняющим словом, а снизу задаваемым ему вопросом от имени поясняемого слова (см. рис. 1). Нумерацией вопросов задаются координаты поясняющих слов в трёхуровневом дереве.

В силу своей наглядности графовая СФ-модель, представляющая отношение пояснения, удобна для анализа поясняющих слов. Слово «скоординированная» само требует пояснения, ибо координация требует взаимодействия нескольких субъектов (не менее двух). Цели деятельности сформулированы как руководство и управление, причём оба они имеют одинаковый состав поясняющих слов-аргументов. Но слова руководство и управление являются синонимами определяемого слова, что порождает два порочных круга в определении понятия «менеджмент качества» по отношению к слову «менеджмент».

\begin{tabular}{|c|c|c|}
\hline$\frac{\text { Деятельность }}{\text { 1. Что это? }}$ & Скоординированная & \multirow{2}{*}{\begin{tabular}{l}
$?$ \\
\hdashline-1. Между кем?
\end{tabular}} \\
\hline \multirow[t]{8}{*}{ 1. Что это? } & 2.1. Какая? & \\
\hline & Руководство & Организация \\
\hline & \multirow[t]{3}{*}{ 2.2. Какая цель? } & 2.2.1. Чем? \\
\hline & & Качество \\
\hline & & 2.2.2. В каком направлении? \\
\hline & Управление & Организация \\
\hline & \multirow[t]{2}{*}{ 2.3. Какая цель? } & 2.3.1. Чем? \\
\hline & & Качество \\
\hline
\end{tabular}

Рис. 1. Графовая СФ-модель определения понятия «менеджмент качества»

Эти слова можно рассматривать не что иное, как варианты перевода иностранного слова на русский язык. Однако определение понятия - это не словарь иностранных слов. Ещё один порочный круг образуется относительно слова «качество» в обеих частях определения (определяемое определяется через себя). Таким образом, приведённое определение понятия «менеджмент качества» следует считать неудовлетворительным.

\section{3. Функциональный подход}

Под функцией слова будем понимать его значение, зависящее от набора существенных признаков (СП), которыми характеризуется обозначаемое им понятие. В определении понятия роль СП играют актанты повествовательного предложения [11]. Функциональный подход к выяснению смысла слова предполагает использование отношения функиияаргумент. В работе [12] рассмотрены следующие виды функциональной зависимости $a=$ $f\left(a_{1}, \ldots, a_{i}, \ldots, a_{n}\right)$ фактора $a$ от факторов $a_{1}, \ldots, a_{i}, \ldots, a_{n}$ : каузативная (связь «причинаследствие»), суммирующая, альтернативная и транзитивная.

Из этих видов функциональной зависимости для объяснения смысла слова наиболее общей представляется суммирующая зависимость $a=f\left(a_{1}, \ldots, a_{i}, \ldots, a_{n}\right)$. В родовидовом определении понятия она реализуется следующим образом. Определяемому понятию $a$ приравнивается понятие более общей категории $a_{1}$, именуемое родовым, которое уточняется видовыми отличиями $a_{2}, \ldots, a_{i}, \ldots, a_{n}$, выделяющими понятие $a$ от других понятий, видовых по отношению к понятию $a_{1}$. Необходимости совпадения всех СП для объяснения смысла слова отвечает функция логического умножения - конъюнкция $(\wedge)$. В словесной форме конъюнкция выражается союзом И. С учётом главенствующей роли СП $a_{1}$ функция $f$ представляется как: 


$$
a=a_{1} \wedge \bigwedge_{i=2}^{n} a_{i}
$$

Пример. Женщина = человек $\wedge$ женский пол.

По числу видовых отличий понятию присваивается ранг. Если ранг родового понятия принять за 0, то ранг видового понятия равен числу видовых отличий. В примере ранг понятия «Женщина» равен 1.

Полноту Ф-модели слова можно определить через достаточный для понимания (выделения от других схожих с ним смыслов) перечень аргументов, за которые принимаются существенные признаки понятия (семантические актанты предложения). Нахождение семантических актантов отличается от синтаксического анализа предложения. Они находятся путём поиска ответов на некоторый набор вопросов. Перечень основных вопросов в английском языке образно выражается шестёркой слов $6 \mathrm{~W}$, содержащих букву W. К ним относятся следующие слова: Who, What, Where, When, Why, How (буква W в конце слова) [13].

Перечень вопросов зависит от категории определяемого слова (предмет, действие). Аргументы определим через их роли в синтезе смысла определяемого понятия, выраженные вопросительными словами. Из этого перечня исключим вопросительные слова, ответы на которые ситуативны, т.е. зависят от ситуации, в которой используется определяемое понятие. К ним относятся вопросы Где? и Когда?.

Для определения понятия, относящегося к категории действия, полным является следующий перечень вопросов: Действие $=\operatorname{Pr}($ Что это?, Кто субъект действия?, Что за объект действия?, Зачем выполняется действие?, Как выполняется действие?).

Определение понятия, относящегося к категории «предмет», должно отвечать на следующие вопросы: Предмет $=\operatorname{Pr}$ (Что это?, Каким свойством обладает?, Откуда произошёл?). Этот перечень достаточен для определения предметов естественного происхождения, целевое назначение которых изначально неизвестно. Для предметов искусственного происхождения этот перечень должен быть дополнен вопросом «Зачем?».

На рис. 2 изображена Ф-модель определения понятия «менеджмент качества», относящегося к категории действия.

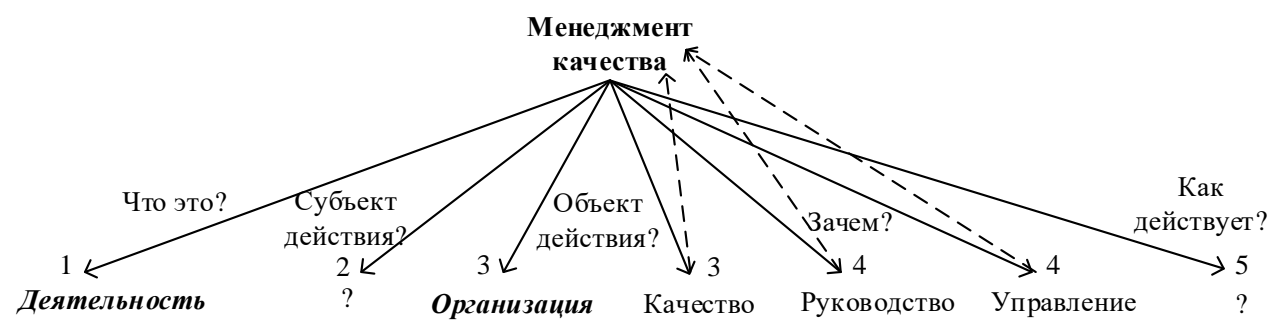

Рис. 2. Функциональная модель определения понятия «менеджмент качества»

Эта модель напрямую связывает определяемый термин с поясняющими его словами в роли существенных признаков понятия. Из трёх обратных связей от СП к термину одна очевидна, поскольку связывает слова «качество». Две других пунктирных связей выявляются через синонимию слов. Отвечает поставленным вопросам только объект деятельности - организация. Прямой ответ на то, кто является субъектом деятельности, отсутствует. По сути, анализируемое определение термина «менеджмент качества» представляет собой некоторый набор ключевых слов, имеющих отношение к определяемому термину. 
Логичным путём к улучшению качества определения является опора на более общее обосновано определённое понятие. Таковым по отношению к слову «менеджмент» является русское слово «управление». В работе [13] оно определено на основе первого закона Ньютона. Его определение отвечает на все вопросы рисунка 2. В той же работе доказана большая общность слова «управление» по отношению к слову «менеджмент». В таком случае в определении термина «менеджмент качества» достаточно конкретизировать цели и средства управления качеством. Для уточнения понятий, относящихся к категории действия, применима О-модель.

\section{4. Операционный подход}

О-модель выражает определяемое действие через элементарные действия, реализуемые во времени и пространстве, называемые в программировании алгоритмом выполнения операции. Применительно к операциям такой перечислительный подход применим к словам, имеющим смысл действия, к которым относятся глаголы и отглагольные существительные. В рассматриваемом примере деятельность в области обеспечения и улучшения качества продукта любого назначения можно описать следующей последовательностью действий:

1) Изучение спроса на продукт;

2) Выбор характеризующих его параметров;

3) Формирование нормативов на значения параметров;

4) Разработка нормативных документов;

5) Организация их выполнения на каждом этапе жизненного цикла продукта;

6) Оценивание соответствия параметров нормативным значениям;

7) Принятие мер по устранению несоответствия;

8) Разработка предложений по повышению качества продукта;

9) Идти к 1.

Этот перечень действий в принципе можно назвать операционным определением понятия «менеджмент качества» с той оговоркой, что перечисленные действия предполагают не только управление ими, но и их исполнение. Однако подробная детализация противоречит требованию краткости определения. При его расширении оно переходит в категорию объяснения. Аналогичный перечислительный подход можно применить к свойствам предмета, назвав его квазиоперационным подходом.

\section{5. Применение предлагаемых подходов в компьютерной лингвистике}

Предлагаемые подходы к оцениванию качества определений понятий имеют прямое отношение к такому разделу компьютерной лингвистики, как компьютерная лексикография. В её рамках решаются такие задачи как определение информативности лексических и словосочетательных единиц, стандартизация и ведение терминосистем, толкование слов в толковых словарях и т.д.

В качестве одного из способов толкования слов в толковых словарях применяются определения понятий. Например, в толковом словаре Ожегова одежда определена как совокупность предметов, которыми покрывают, облекают тело [14]. В этом определении отсутствует ответ на вопрос «Зачем?». Предложенные подходы могут использоваться для оценивания качества подобного рода определений в рамках метода компонентного анализа при выделении минимального набора признаков у определенной совокупности языковых единиц и категорий языка. Они могут оказаться востребованными при обсуждении определений понятий в рамках пополнения русского викисловаря [15].

Одним из разделов википедии [16] является инженерия онтологий - область, в которой изучаются методы и методологии построения онтологий. Онтология - формальное представление набора понятий в предметной области и взаимосвязи между этими 
понятиями. Качество онтологий зависит от смысла употребляемых в них понятий. Предложенные подходы должны способствовать уточнению смысла понятий.

\section{Заключение}

Проблема создания и понимания текстов тесно увязана с правильным подбором используемых слов. Этой цели служат, в частности, словари. Их разновидностью являются толковые словари русского языка. Одним из видов толкования слов являются определение их смысла через слова с известным смыслом. Терминология прикладных наук формируется специалистами в своих областях, не имеющими достаточных лингвистических знаний. Познавательная функция эвристически сформулированных определения понятий часто оказывается неудовлетворительной. Предложенные в работе подходы, основанные на использовании собственных свойств моделей, позволяют формализовать и частично автоматизировать процесс анализа определений понятий. Целью анализа является оценивание качества определений и содержаний понятий на предмет удовлетворения системным требованиям - полноты, неизбыточности, отсутствия порочных циклов.

Структурный подход позволяет найти избыточные и недостающие поясняющие слова в определения понятия, представленного в форме повествовательного предложения. Функциональный подход позволяет оценить полноту содержания понятия и наличие порочных циклов. Операционный подход уточняет понятия, относящиеся к категории действия, через элементарные действия.

Предложенные подходы к анализу определения понятия дополняют друг друга и позволяют рассматривать определение с различных точек зрения. При рассмотрении определения понятия как системы текстового типа предложенные подходы в совокупности позволяют оценить и улучшить его качество. Помимо их применения для улучшения качества профессиональных терминосистем, они могут быть востребованы при решении ряда задач компьютерной лексикографии и покомпонентного анализа.

Исследования, выполненные по данной тематике, проводились при финансовой поддержке грантов РФФИ № 19-08-00989, № 20-08-01046 в рамках бюджетной темы № 0073-2019-0004.

\section{Литература}

1 Полонников Р.И. Избранные труды в двух томах / СПб: «Анатолия». 2013. Т. 1. С. 249.

2 Ермакова А.В. Природа термина // Вестник Нижегородского университета им. Н.И. Лобачевского. 2018. №2. С. 218-223.

3 ГОСТ 20911-89. Техническая диагностика. Основные термины и определения.

4 Лотте Д.С. Основы построения научно-технической терминологии //М.: Изд. АН СССР. $1961.155 \mathrm{c}$.

Целищев В.В. Рационалистический оптимизм и философия Курта Геделя // Вопросы философии. 2013. № 8. С. 12-23.

Микони С.В. Общие диагностические базы знаний вычислительных систем / СПб.: СПИИРАН. 1992. 234 с.

7 Микони С.В., Соколов Б.В. Юсупов Р.М. Квалиметрия моделей и полимодельных комплексов // М.: РАН. 2018. 314 с.

8 Колмогоров А.Н., Драгалин А. Г. Введение в математическую логику // М.: Изд-во МГУ. 1962. 120 с.

9 Курош А.Г. Лекции по общей алгебре // СПб. : Лань. 2007. 556 с. 
[10]ГОСТ ISO 9000-2011. Системы менеджмента качества. Основные положения и словарь.

[11] Теньер Л. Основы структурного синтаксиса. / Пер. с франц. Вступ. ст. и общ. ред. В. Г. Гака // М.: Прогресс, 1988. 656 с.

[12]Микони С.В. Формализованный подход к установлению связи и роли понятий. Компьютерная лингвистика и вычислительные онтологии. Выпуск 2 (Труды XXI Международной объединенной конференции «Интернет и современное общество», IMS-2018. СПб. 30.05-2.06.2018. Сборник научных статей). СПб.: Университет ИТМО. 2018. C. 75-84.

[13] Микони С.В. Обобщённая онтологическая модель управления в концепции социокиберфизической системы // Онтология проектирования. 2019. Т.9, №2(32). С.191-202.

[14] Толковый словарь Ожегова. Электронная версия URL: https://slovarozhegova.ru/ (дата обращения: 07.05.2020).

[15] Викисловарь URL: https://ru.wiktionary.org/wiki/понятие (дата обращения: 07.05.2020).

[16]Википедия URL: https://ru.qwe.wiki/wiki/Ontology_engineering (дата обращения: 07.05.2020).

\title{
Three Approaches to the Definition of Concepts Based on the Model's Own Properties
}

\author{
S. Mikoni
}

St. Petersburg Institute for Informatics and Automation of the Russian Academy of Sciences

The application of a systematic approach to the analysis of the definition of a concept is proposed. It is considered as a text model to which its own properties of model are applicable. These properties are derived from the first-order predicate language model, which is the algebraic structure. The own property of the model includes the function of the object that implements its operation and structure. Each property is represented by an elementary model: functional (Fmodel), operating (O-model) and structural (S-model) model.

The use of these models for analyzing the definition of a concept is called the functional, operational, and structural approach, respectively. The essence of each approach is illustrated by the example of the definition of the term "quality management", taken from the international terminological standard. Visual graph models are used to illustrate approaches.

The structural approach allows you to find redundant and missing explanatory words in the definition of a concept presented in the form of a narrative sentence. The functional approach allows us to assess the completeness of the concept and the presence of vicious cycles. The operational approach clarifies concepts related to the category of action through elementary actions.

Keywords: word, meaning, concept, model, property, basis, function, operation, structure, relation, argument

Reference for citation: Mikoni S.V. Three approaches to the definition of concepts based on the model's own properties // Computer Linguistics and Computing Ontologies. Vol. 4 (Proceedings of the XXIII International Joint Scientific Conference «Internet and Modern Society», IMS-2020, St. Petersburg, June 17-20, 2020). - St. Petersburg: ITMO University, 2020. P. 109 - 117. DOI: 10.17586/0000-0000-2020-4-109-117

\section{References}

[1] Polonnikov R.I. Izbrannye trudy v dvuh tomah // SPb: «Anatoliya». 2013. T. 1. S. 249. [In Russian]. 
2 Ermakova A.V. Priroda termina // Vestnik Nizhegorodskogo universiteta im. N.I. Lobachevskogo. 2018. №2. S. 218-223. [In Russian].

3 GOST 20911-89. Tekhnicheskaya diagnostika. Osnovnye terminy i opredeleniya. [In Russian].

4 Lotte D.S. Osnovy postroeniya nauchno-tekhnicheskoj terminologii // M.: Izd. AN SSSR. 1961. 155 s. [In Russian].

Celishchev V.V. Racionalisticheskij optimizm i filosofiya Kurta Gedelya // Voprosy filosofii. 2013. № 8. S. 12-23. [In Russian].

Mikoni S.V. Obshchie diagnosticheskie bazy znanij vychislitel'nyh sistem // SPb.: SPIIRAN. 1992. 234 s. [In Russian].

7 Mikoni S.V., Sokolov B.V. YUsupov R.M. Kvalimetriya modelej i polimodel'nyh kompleksov // M.: RAN. 2018. 314 s. [In Russian].

8 Kolmogorov A.N., Dragalin A. G. Vvedenie v matematicheskuyu logiku // M.: Izd-vo MGU. 1962. 120 s. (In Russian). [In Russian].

9 Kurosh A.G. Lekcii po obshchej algebre // SPb. : Lan'. 2007. 556 s. [In Russian].

10 GOST ISO 9000-2011. Sistemy menedzhmenta kachestva. Osnovnye polozheniya i slovar'. [In Russian].

11 Ten'er L. Osnovy strukturnogo sintaksisa. / Per. s franc. Vstup. st. i obshch. red. V. G. Gaka // M.: Progress, 1988. 656 s. [In Russian].

12 Mikoni S.V. Formalizovannyj podhod k ustanovleniyu svyazi i roli ponyatij. Komp'yuternaya lingvistika i vychislitel'nye ontologii. Vypusk 2 (Trudy XXI Mezhdunarodnoj ob"edinennoj konferencii «Internet i sovremennoe obshchestvo», IMS-2018. SPb. 30.05-2.06.2018. Sbornik nauchnyh statej). SPb.: Universitet ITMO. 2018. S. 7584 [In Russian].

13 Mikoni S.V. Obobshchyonnaya ontologicheskaya model' upravleniya v koncepcii sociokiberfizicheskoj sistemy // Ontologiya proektirovaniya. 2019. T.9, №2(32). S.191-202. [In Russian].

14 Tolkovyj slovar' Ozhegova. Elektronnaya versiya URL: https://slovarozhegova.ru/ (data obrashcheniya: 07.05.2020). [In Russian].

1 Vikislovar' URL: https://ru.wiktionary.org/wiki/ponyatie (data obrashcheniya: 07.05.2020). [In Russian].

1 Vikipediya URL: https://ru.qwe.wiki/wiki/Ontology_engineering (data obrashcheniya: 07.05.2020). [In Russian]. 